\title{
Leaf Surface Fungi of Early Blight [Alternaria solani (Ellis and Martin)] Infected and Non-Infected Leaves of Tomato [Solanum lycopersicum (L.)]
}

\author{
M. Hajong ${ }^{1 *}$, T. Rajesh ${ }^{1}$, R.K. Tombisana Devi ${ }^{1}$, D. Thakuria ${ }^{2}$, M. Rai ${ }^{3}$, \\ G.T. Behere ${ }^{4}$, H. Rymbai ${ }^{5}$, M. Debbarma ${ }^{1}$ and O.D. Nongthombam ${ }^{1}$
}

${ }^{1}$ School of Crop Protection (SCP), College of Post Graduate Studies (CPGS), Central Agricultural University (CAU), Umiam, Ribhoi-793103, Meghalaya, India

${ }^{2}$ School of Natural Resource Management, CPGS, CAU, Umiam, Ribhoi-793103, Meghalaya, India

${ }^{3}$ School of Crop Improvement, CPGS, CAU, Umiam, Ribhoi-793103, Meghalaya, India ${ }^{4}$ Division of Entomology, Indian Council of Agricultural Research (ICAR) Research Complex for North Eastern Hill Region (RCNEHR), Umiam, Ribhoi-793103, Meghalaya, India ${ }^{5}$ Division of Horticulture, ICAR-RCNEHR, Umiam, Ribhoi-793103, Meghalaya, India

*Corresponding author

\section{A B S T R A C T}

Keywords

Leaf impression method, Leaf surface fungi, Leaf washing and dilution plating method, Leaf washing and serial dilution plating and microbial population

Article Info

Accepted: 07 June 2019 Available Online: 10 July 2019
The tomato leaves infected with early blight recorded the highest leaf surface fungal species than non-infected leaves. In total twenty six fungal species (thirteen genera) were isolated from both diseased infected and noninfected leaf surface of tomato from seven districts of Meghalaya, following leaf impression, leaf washing and dilution plating and leaf washing and serial dilution plating methods. Among all the three methods used leaf impression method recovered the highest fungal population followed by serial dilution plating and leaf washing and dilution plating method. The predominant fungal species found both in infected and noninfected leaves were Fusarium sp., Phoma sp., Penicillium sp., Aspergillus sp. Trichoderma sp. and Chaetomium sp. Whereas, Camarosporum sp., $F$. pallidoroseum and $P$. glabrum were recovered only from healthy leaf samples. The further investigation can be done to find the antagonistic potential of leaf surface fungi against the major foliar diseases of tomato.

\section{Introduction}

Tomato ( $S$. lycopersicum L.) is one of the most popular vegetable crops worldwide which share a great position in India as fresh vegetable. It belongs to the Solanaceae family which originated in the Andean region of South America. It is grown in a wide range of climate and the largest production centres are in southern and central part i.e., Andhra 
Pradesh, Madhya Pradesh, Karnataka, Gujarat, Odissa, West Bengal, Talengana, Chhattisgarh, Maharashtra and Bihar (Horticulture Statistics Division, Department of Agriculture, Cooperation and Farmers' Welfare, 2018). Area under tomato cultivation in the country is about $7.3 \%$ of the total cropped land under vegetables and the area and production during 2017-2018 was 786 hectare (ha) and 19,377 Metric Ton (MT), respectively (Department of Agriculture Cooperation and Farmers Welfare, 2017). The agro-climatic condition of Meghalaya is also favourable for the cultivation of vegetables throughout the year. Among the vegetables, tomato is one of the most popular and widely grown vegetables crop in Meghalaya. In Meghalaya, it is cultivated throughout the year during rainy, winter and summer seasons. It occupies an area of 55.081 thousand ha with production of 35.51 MT during 2017-18 (Horticulture Statistics Division, Department of Agriculture, Cooperation and Farmers' Welfare, 2018).

Leaf surface is a habitat for a variety of microflora including pathogens and saprophytes. Leaf surface fungi are the mycota which grow on the leaf surfaces (Langvad, 1980). These group of fungi are categorized into two groups i.e., casuals and residents (Norse, 1972). Casuals land on the surface of leaves but cannot grow whereas residents can multiply on the healthy leaf surface without noticeably affecting the host (Leben, 1965). Leaf surface fungi have not been fully studied and are still misused especially compared to rhizobacteria, root and seed endophytes and pathogenic microbes. Most of the work on leaf surface fungi was concerned with the pathogens or non-parasitic fungi of economically important trees (Dickinson, 1967; Pugh and Williams, 1968; Lamb and Brown, 1970; Pugh and Mulder, 1971; Bainbridge and Dickinson, 1972; Norse, 1972; Mishra and Dickinson, 1981;
Cabral, 1985). This group of fungi were studied from mangroves (Kuthubutheen, 1981, 1984; Sivakumar and Kathiresan, 1990).

Due to the deposits of nutrients on the leaf surface is a favourable environment for millions of microbes. These microbial communities can be affected by internal and external agents like temperature, humidity, nutrient availability, leaf type and age and the presence of inhibitors (chemical compounds produced by the plant) (Andrews, 1991; Kinkel, 1997; De Jager et al., 2001; Santamaria and Bayman, 2005; Evueh and Ogbebor, 2008). These microbes can either be beneficial or harmful to the host plant. Beneficial microbes cannot cause any disease symptoms unlike phytopathogens (Malfanova et al., 2013). Therefore, leaf surface microflora is essential to study the environmental microbial diversity. It will also suggest the role of such community to the health and wellbeing of the plant as well as on members of the food chain that consume them. The three methods used in this investigation include leaf washing and dilution plating, leaf washing and serial dilution plating and leaf impression for culturing culturable fungi. The present investigation was carried out from one of the important vegetable crops i.e., tomato. Three methods are employed to study leaf surface fungi (Lindsey, 1976).

\section{Materials and Methods}

\section{Sample collection}

Both early blight diseases infected and noninfected leaf samples of tomato were collected from 24 locations from seven districts i.e., Ri-Bhoi, East Khasi Hills, West Jaintia Hills, East Jaintia Hills, North Garo Hills, West Garo Hills and South West Garo Hills of Meghalaya during the tomato 
growing season from 2017-2018. Randomly twenty samples (twenty leaves per sample) from each field were collected in sterile polybags and taken back to laboratory for isolation of leaf surface fungi. Both the healthy and disease infected leaves were handpicked by holding the petiole only and placed into a separate fresh polyethylene bag, respectively. On bringing to the laboratory, department of plant pathology, SCP, CPGS, CAU, Meghalaya samples were immediately transferred to refrigerator $\left(4 \pm 1^{\circ} \mathrm{C}\right)$ until further processing which normally was within 24 hours (h) of collection.

\section{Isolation method}

Three isolation methods were evaluated to characterize and identify the leaf surface fungi of tomato. The leaf surface fungi were isolated by leaf impressions (Dickinson et al., 1974) and modified leaf washing method (Dickinson, 1971).

\section{Leaf Impression method of isolation}

Randomly, ten diseased infected and ten noninfected leaves were collected from the various locations. Collected samples were then rinsed for 10-15 times with tap water followed by sterilized distilled water (SDW) under a laminar air flow hood to remove externally loosely attached dusts and microbes.

The leaf impression was made by pressing both the leaf surfaces (upper and lower, separately) against potato dextrose agar (PDA) (Hi-media Ltd., Mumbai) in petri dishes to produce the leaf-imprints.

After leaving for an hour, leaves were then discarded and the plates were incubated at $28 \pm 1^{\circ} \mathrm{C}$ till colonies grow on the agar surface. The sample from every location was repeated for three times.

\section{Leaf washing method of isolation}

A modified leaf washing method was adopted (Dickinson, 1971) to estimate the leaf surface fungi. To estimate the leaf surface fungi, discs of 0.5 centimetres diameter was cut randomly from the washed tomato leaves with sterile cork borer aseptically. Twenty five discs for both the infected and non-infected leaves were placed separately in $250 \mathrm{ml}$ conical flask containing fifty $\mathrm{ml}$ SDW. Then, the flask was shaken in a shaker for about 20 minutes to get a homogenous suspension of the microbial propagules. Sterilized Petri plates were poured with PDA medium and solidified. Using spread plate method one ml microbial suspension was pipetted out into the agar plates. Plates were then sealed with parafilm and incubated at $28 \pm 1^{\circ} \mathrm{C}$ for three days. Each sample was repeated for three times. Different colonies grown from leaf washes were subcultured, purified and fungi were preserved on PDA slants, respectively, at $4^{\circ} \mathrm{C}$. The total microbial population per square $\mathrm{cm}$ of leaf surface of tomato was calculated by using the following formula (Vimala and Suriachandraselvan, 2006).

Total number of microbes in $1 \mathrm{ml}$ Total number of microbes = ------------ X 100 Total area of 25 discs $\mathrm{x} 2$ (Area of 1 disc $=\pi r^{2}$, where $r$ is the radius of disc in $\mathrm{cm}$ )

\section{Leaf washing and serial dilution plating technique}

From the homogenous stock suspension of the microbial propagules as prepared by the above mentioned leaf washing method, a dilution series was made upto $10^{-5}$ of each sample suspension (healthy and infected stock suspension). One $\mathrm{ml}$ aliquots of the $10^{-3}$ dilutions were surface plated in triplicate on PDA media containing petri plates (de Jager et al., 2001). 
Identification and characterization of fungal isolates

Fungal colonies were subcultured after 3-4 days of incubation and pure cultures were transferred to PDA slants. Fungal isolates were studied based on cultural, morphological (Domsch et al., 1980; Kharwar et al., 2012) and microscopic characteristics viz. mycelium, conidiophore, spore structure etc. under microscope (Leica ICC50, Germany) by cover slip insertion method. And also those unidentified isolates were sent to National Centre of Fungal Taxonomy (NCFT), New Delhi for identification up to species level.

\section{Results and Discussion}

\section{Isolation and identification}

The diseased infected and non-infected leaf samples were collected from 24 locations from seven districts of Meghalaya. Altogether, twenty six fungal species of thirteen genera were isolated and identified on the basis of colony morphology, mycelia, sporangiophore and spore structure.

The predominant fungal species found both in infected and non-infected leaves were Fusarium sp., Phoma sp., Penicillium sp., Aspergillus sp. Trichoderma sp. and Chaetomium sp. Whereas, Camarosporum sp., F. pallidoroseum and $P$. glabrum were recovered only from the non-infected leaf samples (Table 1).

The genus Phoma recovered the total of six species followed by Trichoderma which is four species and Fusarium and Penicillium were three species.

The highest fungal isolates were recovered from the class Dothideomycetes i.e., five genus and ten species. And least fungal isolates were recovered from the class Mucoromycotina and Oomycota i.e., one genus and one species.

Among all the three methods used in this study, leaf impression infected upper leaves recovered the highest fungal population i.e., $1.9 \times 10^{3}$ microbial population $/ \mathrm{cm}^{2}$ whereas the lowest fungal population was recovered from dilution plating healthy leaves i.e., $6.2 \mathrm{X}$ $10^{2}$ microbial population $/ \mathrm{cm}^{2}$ (Table 2).

Though leaf impression method recovered higher fungal flora but it was difficult to assess the microbes quantitatively as very high density population was recovered. Also the microbial counting was difficult due to mixed nature of microbial population for their competition of nutrition and space. But, leaf impression method was a quick and simple method of isolation of leaf surface microflora (Dickinson, 1971).

Infected upper leaf surface recovered more fungal isolates than healthy leaf surface. Compared to all the three methods employed, leaf washing and dilution plating method was the most efficient method as it enabled the isolation of greater number and higher recovery of the same species. The morphological and cultural characteristics of all the fungal isolates were given in table 3 .

Plant surfaces or internal tissues play as a home or niche for microorganisms. Therefore, plant surfaces are noted as a vital environment for microorganisms based on either a permanent (resident's epiphytes, endophytes or pathogens) and transient (unspecific epiphytic saprophytes) association (Forseca and Inacio, 2006).

In aerial leaf surface many organisms are found common in several crop plants. Only some species are restricted to a particular crop plant. 
Table.1 Presence (+) or absence (-) of fungi isolated from diseased infected (I) and non-infected (NI) leaves of Tomato

\begin{tabular}{|c|c|c|c|c|c|c|c|c|c|}
\hline \multirow{4}{*}{$\begin{array}{l}\text { Sr. } \\
\text { No. }\end{array}$} & \multirow[t]{4}{*}{ Name of the fungi } & \multicolumn{8}{|c|}{ Isolation Methods } \\
\hline & & \multicolumn{2}{|c|}{$\begin{array}{l}\text { Leaf washing } \\
\text { and dilution } \\
\text { plating }\end{array}$} & \multicolumn{2}{|c|}{$\begin{array}{l}\text { Leaf washing } \\
\text { and Serial } \\
\text { dilution plating }\end{array}$} & \multicolumn{4}{|c|}{ Leaf Impression } \\
\hline & & \multirow[t]{2}{*}{ I } & \multirow[t]{2}{*}{ NI } & \multirow[t]{2}{*}{ I } & \multirow{2}{*}{ NI } & \multicolumn{2}{|c|}{ I } & \multicolumn{2}{|c|}{ NI } \\
\hline & & & & & & $\mathbf{U}$ & $\mathbf{L}$ & $\mathbf{U}$ & $\mathbf{L}$ \\
\hline 1 & Alternaria alternata & + & - & - & - & + & - & - & - \\
\hline 2 & Botryodiplodia theobromae & - & - & - & - & + & - & + & - \\
\hline 3 & Camarosporum species novo. & - & - & - & - & - & - & - & + \\
\hline 4 & Cladosporium cladosporioides & - & + & - & - & + & - & + & - \\
\hline 5 & Phoma glomerata & - & + & + & - & - & + & + & + \\
\hline 6 & P. sorghina & - & + & - & - & - & - & + & - \\
\hline 7 & P. lingam & - & - & - & - & - & - & + & - \\
\hline 8 & P. exigua & + & - & - & - & + & - & + & - \\
\hline 9 & P. herbarum & - & - & + & - & + & + & - & - \\
\hline 10 & P. terrestris & - & - & - & - & + & - & + & - \\
\hline 11 & Aspergillus niger & + & - & + & - & - & + & - & + \\
\hline 12 & A. flavus & + & + & - & - & - & - & + & - \\
\hline 13 & Penicillium sp. & + & - & - & - & - & - & - & + \\
\hline 14 & Penicillium sp. & + & - & + & - & - & - & - & + \\
\hline 15 & P. glabrum & - & - & - & - & - & - & + & - \\
\hline 16 & Rhizopus sp. & + & - & + & + & + & - & - & + \\
\hline 17 & Pythium aphanidermatum & + & + & + & - & + & - & + & - \\
\hline 18 & Acremonium strictum & - & - & - & - & + & + & + & + \\
\hline 19 & Chaetomium globosum & - & + & - & + & - & - & + & + \\
\hline 20 & Fusarium pallidoroseum & - & - & - & - & - & - & - & + \\
\hline 21 & F. oxysporum & + & - & + & - & + & - & + & - \\
\hline 22 & F. solani & + & - & - & - & - & + & + & - \\
\hline 23 & Trichoderma sp. & - & - & - & - & - & - & + & - \\
\hline 24 & T. harzianum & - & + & - & + & - & - & - & - \\
\hline 25 & T. viride & - & + & + & + & - & - & - & - \\
\hline 26 & T. asperullum & - & + & - & + & - & - & + & - \\
\hline
\end{tabular}

Table.2 Total microbial population per square $\mathrm{cm}$ of leaf surface of tomato [counts in colony forming units per millitre/leaf $(\mathrm{cfu} / \mathrm{ml})]$

\begin{tabular}{|l|l|c|}
\hline Sr. No. & Methods & Mean fungal population in $\mathbf{1 ~ m l ~}\left(\mathbf{c m}^{-\mathbf{2}}\right)$ \\
\hline $\mathbf{1}$ & Leaf impression-healthy upper leaves & $1.1 \times 10^{3}$ \\
\hline $\mathbf{2}$ & Leaf impression-healthy lower leaves & $9.9 \times 10^{2}$ \\
\hline $\mathbf{3}$ & Leaf impression-infected upper leaves & $1.9 \times 10^{3}$ \\
\hline $\mathbf{4}$ & Leaf impression-infected lower leaves & $1.3 \times 10^{3}$ \\
\hline $\mathbf{5}$ & Dilution plating-healthy leaves & $6.2 \times 10^{2}$ \\
\hline $\mathbf{6}$ & Dilution plating-infected leaves & $1.3 \times 10^{3}$ \\
\hline $\mathbf{7}$ & Serial Dilution plating-healthy leaves & $7.6 \times 10^{2}$ \\
\hline $\mathbf{8}$ & Serial Dilution plating-infected leaves & $1.7 \times 10^{3}$ \\
\hline
\end{tabular}


Table.3 Morphological and cultural characteristics of leaf surface fungal isolates of tomato

\begin{tabular}{|c|c|c|c|c|c|c|c|c|c|c|}
\hline \multirow{3}{*}{$\begin{array}{l}\text { Sr. } \\
\text { No. }\end{array}$} & \multirow{3}{*}{$\begin{array}{l}\text { Fungal } \\
\text { isolates }\end{array}$} & \multirow[t]{3}{*}{ Class } & \multicolumn{5}{|c|}{ Cultural characteristics } & \multicolumn{2}{|c|}{ Microscopic observation } & \multirow[t]{2}{*}{ Nutrition } \\
\hline & & & \multicolumn{2}{|c|}{ Colour of isolate } & \multirow{2}{*}{$\begin{array}{l}\text { Growth } \\
\text { rate }\end{array}$} & \multirow{2}{*}{$\begin{array}{l}\text { Text } \\
\text { ure }\end{array}$} & \multirow[t]{2}{*}{ Margin } & \multirow[t]{2}{*}{ Mycelium and Conidiophore } & \multirow[t]{2}{*}{ Conidia (Shape and colour) } & \\
\hline & & & Front & Reverse & & & & & & \\
\hline 1 & A. alternata & Dothideomycetes & $\begin{array}{l}\text { Dark } \\
\text { black }\end{array}$ & $\begin{array}{l}\text { Dark } \\
\text { black }\end{array}$ & S & $P$ & $\mathrm{Cr}$ & $\begin{array}{l}\text { Septate and branched brown colour } \\
\text { hyphae. Pale brown straight } \\
\text { conidiophores containing long chain } \\
\text { conidia }\end{array}$ & $\begin{array}{l}\text { Large, dark brown obclavate } \\
\text { conidia in chain with short } \\
\text { conical beak at the tip }\end{array}$ & Saprophyte \\
\hline 2 & B. theobromae & do & $\begin{array}{l}\text { Greyish } \\
\text { black }\end{array}$ & $\begin{array}{l}\text { Dark } \\
\text { black }\end{array}$ & $\mathrm{F}$ & $\mathrm{C}$ & I & $\begin{array}{l}\text { Conidiophores are hyaline, simple, } \\
\text { sometimes septate, rarely branched } \\
\text { cylindrical and arising from the inner } \\
\text { layers of cells lining the pycnidial } \\
\text { cavity. Pycnidia dark black colour }\end{array}$ & $\begin{array}{l}\text { Immature conidia whitish } \\
\text { with thin walls and mature } \\
\text { conidia dark brown with } \\
\text { septa and thick walls }\end{array}$ & do \\
\hline 3 & $\begin{array}{l}\text { Camarosporum } \\
\text { species novo. }\end{array}$ & do & $\begin{array}{l}\text { Greyish } \\
\text { black }\end{array}$ & $\begin{array}{l}\text { Greyish } \\
\text { black }\end{array}$ & $\mathrm{F}$ & $\mathrm{C}$ & $\mathrm{Cr}$ & $\begin{array}{l}\text { Septate and dark coloured mycelium. } \\
\text { Conidiomata pycnidial }\end{array}$ & $\begin{array}{l}\text { Conidia hyaline, aseptate and } \\
\text { subcylindrical }\end{array}$ & do \\
\hline 4 & $\begin{array}{l}C . \\
\text { cladosporioides }\end{array}$ & do & $\begin{array}{l}\text { Dark } \\
\text { grey }\end{array}$ & $\begin{array}{l}\text { Dark } \\
\text { black }\end{array}$ & M & $\mathrm{V}$ & $\mathrm{Cr}$ & $\begin{array}{l}\text { Branched hyphae. Cylindrical } \\
\text { branched conidiophore }\end{array}$ & $\begin{array}{l}\text { Dark, single celled lemon } \\
\text { shaped conidia }\end{array}$ & do \\
\hline 5 & P. glomerata & do & $\begin{array}{l}\text { Pale } \\
\text { pink and } \\
\text { greyish } \\
\text { centre }\end{array}$ & $\begin{array}{l}\text { Pale } \\
\text { pink }\end{array}$ & $\mathrm{F}$ & WC & $\mathrm{Cr}$ & $\begin{array}{l}\text { Dark brown septate hyphae. } \\
\text { Chlamydospores in branched or un- } \\
\text { branched chains. Chlamydospores } \\
\text { showed both longitudinal and } \\
\text { transverse septations as in commonly } \\
\text { seen in the genus Alternaria. }\end{array}$ & $\begin{array}{l}\text { Single celled hyaline, ovoid to } \\
\text { ellipsoidal conidia. Conidia are } \\
\text { bi-gulttulate (containing } 2 \text { oil } \\
\text { drplets) }\end{array}$ & do \\
\hline 6 & P. sorghina & do & $\begin{array}{l}\text { White } \\
\text { later } \\
\text { greyish }\end{array}$ & Greyish & $\mathrm{F}$ & $\mathrm{C}$ & $\mathrm{Cr}$ & $\begin{array}{l}\text { Hyphae septate and hyaline. } \\
\text { Chlamydospores are unicellular, dark } \\
\text { brown and botryoid-alternarioid shape. } \\
\text { Pycnidia grey, globose and ostiolate }\end{array}$ & $\begin{array}{ll}\text { Conidia } & \text { ellipsoidal to } \\
\text { cylindrical, } & \text { smooth, hyaline } \\
\text { and aseptate } & \end{array}$ & do \\
\hline 7 & P. lingam & do & Whitish & Whitish & $\mathrm{F}$ & WC & $\mathrm{Cr}$ & $\begin{array}{l}\text { Hyphae septate and hyaline. Pycnidia } \\
\text { dark, globose and ostiolate }\end{array}$ & $\begin{array}{l}\text { Conidia ellipsoidal, smooth, } \\
\text { hyaline and single celled }\end{array}$ & do \\
\hline 8 & P. exigua & do & $\begin{array}{l}\text { Pale } \\
\text { pink }\end{array}$ & $\begin{array}{l}\text { Pale } \\
\text { pink }\end{array}$ & $\mathrm{F}$ & $\mathrm{C}$ & $\mathrm{Cr}$ & $\begin{array}{l}\text { No chlamydospores, dark walled } \\
\text { pycnidia }\end{array}$ & $\begin{array}{l}\text { Oblong to elliptic or often } \\
\text { irregular hyaline conidia }\end{array}$ & do \\
\hline 9 & P. herbarum & do & $\begin{array}{l}\text { Whitish } \\
\text { grey }\end{array}$ & Greyish & $\mathrm{F}$ & $\mathrm{C}$ & I & $\begin{array}{l}\text { No chlamydospores, dark walled } \\
\text { pycnidia, ostioles often with short } \\
\text { beaks }\end{array}$ & $\begin{array}{l}\text { Oblong to cylindrical with } \\
\text { rounded ends hyaline conidia }\end{array}$ & do \\
\hline 10 & P. terrestris & do & $\begin{array}{l}\text { Pale } \\
\text { pink }\end{array}$ & Reddish & $\mathrm{F}$ & $\mathrm{C}$ & $\mathrm{Cr}$ & $\begin{array}{l}\text { Mycelium hyaline and septate. } \\
\text { Pycnidia dark brown, subglobose, } \\
\text { ostiolate and occur singly. }\end{array}$ & $\begin{array}{l}\text { Conidia oblong to ovoid, } \\
\text { hyaline and aseptate }\end{array}$ & do \\
\hline 11 & A. niger & Eurotiomycetes & $\begin{array}{l}\text { Dark } \\
\text { black }\end{array}$ & $\begin{array}{l}\text { Pale } \\
\text { white }\end{array}$ & $\mathrm{F}$ & $P$ & I & $\begin{array}{l}\text { Mycelium septate, branched and } \\
\text { hyaline. Erect, unbranched, single and } \\
\text { club shaped conidiophore }\end{array}$ & $\begin{array}{l}\text { Round shaped black coloured } \\
\text { conidia, arranged in a long } \\
\text { chain and single-celled }\end{array}$ & do \\
\hline 12 & A. flavus & do & Greenish & $\begin{array}{l}\text { Pale } \\
\text { white }\end{array}$ & $\mathrm{F}$ & $P$ & I & do & $\begin{array}{l}\text { Round shaped green coloured } \\
\text { conidia, arranged in a long } \\
\text { chain and single celled }\end{array}$ & do \\
\hline 13 & Penicillium sp. & do & $\begin{array}{l}\text { Olive } \\
\text { green } \\
\text { with } \\
\text { concentr } \\
\text { ic circle }\end{array}$ & $\begin{array}{l}\text { Creamis } \\
\mathrm{h}\end{array}$ & $\mathrm{S}$ & FV & I & $\begin{array}{l}\text { Hyphae septate, branched and hyaline. } \\
\text { Erect, unbranched and septate } \\
\text { conidiophore }\end{array}$ & $\begin{array}{l}\text { One-celled hyaline and globose } \\
\text { conidia }\end{array}$ & do \\
\hline 14 & Penicillium sp. & do & $\begin{array}{l}\text { Light } \\
\text { blue }\end{array}$ & $\begin{array}{l}\text { Creamis } \\
\mathrm{h} \text { with } \\
\text { reddish } \\
\text { pigments }\end{array}$ & $S$ & FV & I & do & do & do \\
\hline 15 & P. glabrum & do & $\begin{array}{l}\text { Brownis } \\
\mathrm{h}\end{array}$ & $\begin{array}{l}\text { Brownis } \\
\mathrm{h}\end{array}$ & $\mathrm{F}$ & FV & I & do & do & do \\
\hline 16 & Rhizopus sp. & Mucoromycotina & Greyish & Greyish & $\mathrm{F}$ & $\mathrm{CC}$ & $\mathrm{Cr}$ & $\begin{array}{l}\text { Coenocytic with branched hyphae. } \\
\text { Sporangia are supported by a large } \\
\text { apophysate columella atop a long stalk, } \\
\text { the sporangiophore. Sporangiophores } \\
\text { arise from root-like rhizoids. }\end{array}$ & $\begin{array}{l}\text { Sporangiospores globose, } \\
\text { brown coloured and one-celled. }\end{array}$ & do \\
\hline 17 & $\begin{array}{l}P . \\
\text { aphanidermatum }\end{array}$ & Oomycota & White & White & $\mathrm{F}$ & $\mathrm{CF}$ & $\mathrm{Cr}$ & Coenocytic and hyaline hyphae & $\begin{array}{l}\text { Oogonia terminal, globose and } \\
\text { smooth; antheridia intercalary, } \\
\text { thick walled oospores and } \\
\text { lobed sporangia }\end{array}$ & $\begin{array}{l}\text { Facultative } \\
\text { parasite }\end{array}$ \\
\hline 18 & A. strictum & Sordariomycetes & White & $\begin{array}{l}\text { Pale } \\
\text { white }\end{array}$ & M & $\mathrm{C}$ & I & $\begin{array}{l}\text { Hyphae are hyaline and produce } \\
\text { mostly simple awl-shaped erect } \\
\text { phialides with inconspicuous } \\
\text { collarettes }\end{array}$ & $\begin{array}{l}\text { Conidia hyaline, cynlindrical } \\
\text { and single celled in chains or in } \\
\text { conidial masses arised from } \\
\text { short unbranched single } \\
\text { phialides }\end{array}$ & Saprophyte \\
\hline 19 & C. globosum & do & $\begin{array}{l}\text { Olive } \\
\text { green } \\
\text { but later }\end{array}$ & $\begin{array}{l}\text { Olive } \\
\text { green }\end{array}$ & $\mathrm{F}$ & $P$ & $\mathrm{Cr}$ & $\begin{array}{l}\text { Mycelium often grows in } \\
\text { conglomerate masses that resemble } \\
\text { ropes. Ostiolar dark perithecia with }\end{array}$ & $\begin{array}{l}\text { Flat lemon-shaped and olive } \\
\text { brown ascospores within } \\
\text { clavate ascomata }\end{array}$ & do \\
\hline
\end{tabular}




\begin{tabular}{|c|c|c|c|c|c|c|c|c|c|c|}
\hline & & & $\begin{array}{l}\text { stage } \\
\text { become } \\
\text { dark in } \\
\text { colour }\end{array}$ & & & & & unbranched radiating hairs & & \\
\hline 20 & F. pallidoroseum & do & $\begin{array}{l}\text { Yellowis } \\
\mathrm{h}\end{array}$ & $\begin{array}{l}\text { Yellowis } \\
\mathrm{h}\end{array}$ & $\mathrm{F}$ & $\mathrm{C}$ & I & $\begin{array}{l}\text { Mycelium septate and branched. } \\
\text { Phialides are present }\end{array}$ & $\begin{array}{l}\text { Macroconidia with } 3 \text { septa and } \\
\text { microconida single-celled. } \\
\text { Chlamydospores are present }\end{array}$ & do \\
\hline 21 & F. oxysporum & do & $\begin{array}{l}\text { White } \\
\text { later } \\
\text { become } \\
\text { purplish }\end{array}$ & Purplish & M & $\mathrm{C}$ & I & $\begin{array}{l}\text { Septate and branched mycelium. } \\
\text { Conidiophores were elongated and } \\
\text { sparsely branched }\end{array}$ & $\begin{array}{l}\text { Macroconidia fusiform, slightly } \\
\text { curved, pointed at tip and } \\
\text { mostly three celled. Abundant } \\
\text { microconidia, never in chains, } \\
\text { mostly non-septate and } \\
\text { ellipsoidal. Chlamydospores } \\
\text { are also present }\end{array}$ & do \\
\hline 22 & F. solani & do & White & $\begin{array}{l}\text { Off } \\
\text { white }\end{array}$ & M & $\mathrm{C}$ & I & $\begin{array}{l}\text { Phialides are present. Chlamydospores } \\
\text { are also common }\end{array}$ & $\begin{array}{l}\text { Macroconidia are slightly } \\
\text { curved, hyaline and broad. } \\
\text { Microconidia cylindrical, } \\
\text { hyaline and smooth and } \\
\text { aseptate }\end{array}$ & do \\
\hline 23 & $\begin{array}{l}\text { Trichoderma } \\
\text { sp. }\end{array}$ & do & $\begin{array}{l}\text { Yellowis } \\
\mathrm{h} \text { green }\end{array}$ & $\begin{array}{l}\text { Creamis } \\
\mathrm{h}\end{array}$ & $\mathrm{F}$ & $\mathrm{P}$ & $\mathrm{Cr}$ & $\begin{array}{l}\text { Septate Branched with flask shaped } \\
\text { phialides }\end{array}$ & $\begin{array}{l}\text { Ellipsoidal, typically smooth } \\
\text { with smooth walled conidia. } \\
\text { Green coloured }\end{array}$ & do \\
\hline 24 & T. harzianum & do & Green & $\begin{array}{l}\text { Pale } \\
\text { white }\end{array}$ & $\mathrm{F}$ & $\mathrm{P}$ & $\mathrm{Cr}$ & $\begin{array}{l}\text { Flask shaped phialides and arranged in } \\
\text { divergent groups of } 2-4\end{array}$ & Globose conidia & do \\
\hline 25 & T. viride & do & Green & $\begin{array}{l}\text { Pale } \\
\text { white }\end{array}$ & $\mathrm{F}$ & $\mathrm{P}$ & $\mathrm{Cr}$ & do & do & do \\
\hline 26 & T. asperullum & do & Green & $\begin{array}{l}\text { Pale } \\
\text { white }\end{array}$ & $\mathrm{F}$ & $\mathrm{P}$ & $\mathrm{Cr}$ & do & do & do \\
\hline
\end{tabular}

Note: S: slow, F: fast, M: medium, Cr: circular, I: irregular, P: powdery, C: cottony, V: velvety, FV: flat and velvety, WC: wooly colonies, CC: cotton candy; CF: cottony and fluffy

In this present investigation total twenty six fungal species from thirteen genera were recovered from three different isolation methods. It was found that the composition of tomato leaf surface fungi showed some similarities to other plant species like Egyptian wheat (Mazen et al., 1985), Spinacia oleracea (Singh et al., 1986), Capsicum annuum (Basha et al., 2010), Persea bombycina (Bhuyan et al., 2013) and Abelmoschus esculentus (Ogwu and Osawaru, 2014). The leaf impression method was the quickest and simplest method for the isolation of leaf surface microflora. But counting of colonies was also very difficult because of mixed nature of microbial population; may be slow growing colonies were hidden by fast growing microbes (Gunasekera, 1994).

\section{Acknowledgements}

The authors are thankful to the Dean, CPGS, CAU, Umiam and In-charge, SCP, CPGS, CAU, Umiam, Meghalaya for providing all the necessary facilities throughout the course of investigation. We also thank NCFT, New Delhi for culture identification.

\section{References}

Andrews, J. H., 1991. Future Research Directions in Phyllosphere Ecology. In: Andrews, J. H., and Hirano, S. S. (Ed.), Microbial Ecology of Leave, New York, pp. 467-479.

Bainbridge, A., and Dickinson, C.H., 1972. Effect of fungicides on the microflora of potato leaves. Trans. Br. Mycol. Soc. $59,31-41$.

Basha, H., Hemannavar, V., Ramanujam, B., Rangeshwaran, R., and Sriram, S., 2010. Screening of chilli microflora and other biocontrol agents for their antagonistic effects on Colletotrichum spp. infecting chillies. JPPS 2, 38-44.

Bhuyan, P. M., Sandilya, S. P., and Gogoi, D. K., 2013. Phyllosphere Microflora of Muga Silkworm Host Plant Persea bombycina Kost (Som) Leaves in Jorhat District of Assam, India. Int. Res. J. Biological Sci. 2, 60-65.

Cabral, D., 1985. Phyllosphere of Eucalyptus viminalis: dynamics of fungal populations. Trans. Br. Mycol. Soc. 85, 501-511. 
De Jager, E.S., Wehner, F. C., and Korsten, L., 2001. Microbial ecology of the mango phylloplane. Microb. Ecol. 42, 201-207.

Department of Agriculture Cooperation and Farmers Welfare. 2017. Area and Production of Horticulture Crops - All India: 2016-17 to 2017-18 (Third Advance Est.). http://nhb.gov.in/statistics/StateLevel/2017-18(1st\%20Adv.\%20Est).

Dickinson, C. H., Watson, J., and Wallace, B., 1974. An impression method for examining epiphytic micro-organisms and its application to phylloplane studies. Trans. Br. Mycol. Soc. 63, 616619.

Dickinson, C.H., 1967. Fungal colonization of Pisum leaves. Can. J. Bot. 45, 915-927.

Dickinson, C.H., 1971. Cultural studies of leaf saprophytes. In: Preece, T.F., and Dickinson, C.H. (Ed.), Ecology of leaf surface microorganisms, Academic Press, London.

Domsch, K.H., Games, W., and Anderson, T.H., 1980. Compendium of Soil Fungi. Academic Press, London, pp. 1-860.

Evueh, G.A., and Ogbebor, N.O., 2008. Use of phylloplane fungi as biocontrol agent against Colletotrichum leaf disease of rubber (Hevea brasiliensis Muell. Arg). J. Afr. Biotech. 7, 2569-2572.

Forseca, A., Inacio, J. (2006): Chapter 13: Phylloplane Yeast. In: Peter, G., and Rosa, C. (Ed.), Biodiversity and Ecophysiology of yeast: The Yeast Handbook Series, Springer-Veerlag, Berlin, Germany.

Gunasekera, T.S., 1994. Isolation and enumeration of microbial inhabitants of Tea phylloplane. S.L.J. Tea Sci. 63, 1927.

Horticulture Statistics Division, Department of Agriculture, Cooperation and Farmers' Welfare, Ministry of Agriculture and Farmers' Welfare
Government of India 2018. Horticultural Statistics at a Glance, pp. 1-490.

Kharwar, R. N., Gond, S., Kumar, A., and Mishra, A., 2012. A comparative study of endophytic and epiphytic fungal association with leaf of Eucalyptus citriodora Hook., and their antimicrobial activity. World. J. Microbiol. Biotechnol. 11, 1941-1948.

Kinkel, L.L., 1997. Microbial Population Dynamics on Leaves. Annu. Rev. Phytopathol. 35, 327-347.

Kuthubutheen, A.J., 1981. Fungi associated with the aerial parts of Malaysian mangrove plants. Mycopathologia 76, 33-43.

Kuthubutheen, A.J., 1984. Leaf surface fungi associated with Avicennia alba and Rhizophora mucronata in Malaysia. In: Proceedings of the Asian symposium on mangrove environment - research and management, Kuala Lumpur, Malaysia.

Lamb, R.J., and Brown, J.F., 1970. Nonparasitic microflora on leaf surfaces of Paspalum dilatatum, Salix babylonica and Eucalyptus stellulata. Trans. Br. Mycol. Soc. 55, 383-390.

Langvad, F., 1980. A simple and rapid method for qualitative and quantitative study of the fungal flora of leaves. Can. J. Bot. 26, 666-670.

Leben, C., 1965. Epiphytic micro-organisms in relation to plant diseases. Annu. Rev. Phytopathol. 2, 209-230.

Lindsey, B.I., 1976. A survey of methods used in the study of microfungal succession on leaf surfaces. In: Dickinson, C.H., and Preece, T.F. (Ed.), Microbiology of aerial plant surfaces, Academic Press, London.

Malfanova, N., Lugtenberg, B., and Berg, G., 2013. Bacterial endophytes: who and where, and what are they doing there? Leiden University Repository. Available online as a pdf on.). 
http://hdl.handle.net/1887/20732.2013: 25.

Mazen, M.B., Abdel-Hafez, S.I.I., and Shaban, G.M., 1985. Seasonal fluctuations of phyllosphere and phylloplane fungi of Egyptian wheat. Acta Mycol. 21, 109-116.

Mishra, R.R., and Dickinson, C.H., 1981. Phylloplane and litter fungi of Ilex aquifolium. Trans. Br. Mycol. Soc. 77, 329-337.

Norse, D., 1972. Fungal populations of tobacco leaves and their effect on the growth of Alternaria longipes. Trans. Br. Mycol. Soc. 59, 261-271.

Ogwu, M.C., and Osawaru, M.E., 2014. Comparative Study of microflora population on the phylloplane of common okra [Abelmoschus esculentus 1. (Moench.)]. Nig J. Biotech. 28, 1725.

Pugh, G.J.F., and Mulder, J.L., 1971. Mycoflora associated with Typha latifolia. Trans. Br. Mycol. Soc. 57, 273-282.

Pugh, G.J.F., and Williams, G.M., 1968. Fungi associated with Salsola kali. Trans. Br. Mycol. Soc. 51, 389-396.

Santamaria, J., and Bayman, P., 2005. Fungal epiphytes and endophytes of Coffee leaves (Coffea arabica). Microb. Ecol. $50,1-8$.

Singh, P.N., Sindhu, I.R., and Gupta, K., 1986. Comparative study of phylloplane Mycroflora of Cercospora beticola infected and non-infected leaves of Spinacia oleracea and factors affecting disease severity. PINSA, 689-694.

Sivakumar, A., and Kathiresan, K., 1990. Phylloplane fungi from mangroves. Indian J. Microbiol. 30, 229-231.

Vimala, R., and Suriachandraselvan, M., 2006. Phylloplane microflora of bhendi. Internat. J. agric. Sci. 2, 517-518.

\section{How to cite this article:}

Hajong, M., T. Rajesh, R.K. Tombisana Devi, D. Thakuria, M. Rai, G.T. Behere, H. Rymbai, M. Debbarma and Nongthombam, O.D. 2019. Leaf Surface Fungi of Early Blight [Alternaria solani (Ellis and Martin)] Infected and Non-Infected Leaves of Tomato [Solanum lycopersicum (L.)]. Int.J.Curr.Microbiol.App.Sci. 8(07): 521-529.

doi: https://doi.org/10.20546/ijcmas.2019.807.064 